Check for updates

Cite this: RSC Adv., 2019, 9, 17417

Received 1st April 2019

Accepted 27th May 2019

DOI: $10.1039 / c 9 r a 02451 d$

rsc.li/rsc-advances

\title{
Plasma surface-modification of cellulose nanocrystals: a green alternative towards mechanical reinforcement of $\mathrm{ABS} \uparrow$
}

\author{
Andrés Alanis, (D) Josué Hernández Valdés, ${ }^{a}$ Neira-Velázquez María Guadalupe, ${ }^{a}$ \\ Ricardo Lopez, ${ }^{a}$ Ricardo Mendoza, ${ }^{a}$ Aji P. Mathew, (D) ${ }^{b}$ Ramón Díaz de León*a \\ and Luis Valencia (D)*b
}

\begin{abstract}
This article proposes a strategy to functionalize cellulose nanocrystals by means of plasma-surface modification utilizing monomers of different nature: caprolactone, styrene and farnesene. The surface characteristics of the nanocrystals were studied by different techniques including XPS, FTIR and STEM, demonstrating that this technique allows a successful functionalization, yielding homogenous functionalization which does not alter the rod-like shape of the nanocrystals, and therefore their anisotropic behavior. We have furthermore studied the employment of the modified nanocrystals as reinforcement additive of ABS, which significantly enhanced the impact resistance of the thermoplastic, which could have great implications for industrial applications.
\end{abstract}

\section{Introduction}

Due to the current environmental issues, one of the fundamental questions facing research nowadays is to provide affordable and scalable pathways towards sustainable development. In this context, renewable resources are attractive alternatives to partially-substitute petroleum-based polymers in industrial and domestic applications.

Nanocellulose, isolated through a top-down approach from different biomass sources is a prime alternative in terms of sustainability due to its low cost and abundant sources (coming from the most abundant organic polymer on earth, cellulose), as well as advantageous mechanical properties, low density, non-toxicity, versatility for surface modification and the possibility to generate energy without residue after burning at the end of their life cycle. ${ }^{1,2}$ Nevertheless, for certain applications such as mechanical reinforcement of polymeric materials, nanocellulose has some drawbacks such as a limited compatibility with non-polar matrixes, resulting in detrimental mechanical properties. ${ }^{1,3}$ To overcome these challenges, several strategies for the surface modification of cellulose nanocrystals have been proposed during the last decade, among them for instance polymer grafting through controlled/living radical polymerization techniques have been the most extensively

${ }^{a}$ Research Center for Applied Chemistry, Blvd Enrique Reyna 140, 25253, Saltillo, Mexico

${ }^{b}$ Division of Materials and Environmental Chemistry, Stockholm University, Frescativägen 8, 10691, Stockholm, Sweden. E-mail: luis.alexandro@mmk.su.se

$\dagger$ Electronic supplementary information (ESI) available. See DOI: 10.1039/c9ra02451d studied including ATRP (atom transfer reversible additionfragmentation chain transfer) ${ }^{4-7}$ and RAFT (reversible addition-fragmentation chain transfer) ${ }^{5}$ polymerizations. However, these techniques are still very limited in terms of scalability due to the large reaction times that usually require, and in terms of environmental friendliness, as they usually require the use of initiators, and organic solvents, besides of extensive purification steps post-modification.

Plasma-induced polymerization is a simple, efficient and replicable method in which a nanometric layer of polymer is deposited on the surface of nanoparticles, thus significantly tuning their surface chemistry. The main principle of this technique is the ionization of molecules and generation of free radicals through electric glow discharge. ${ }^{8-10}$ The polymerization-initiating species are formed on the surface that get contact with plasma, and the growth mechanism of the polymer chains is through free-radical chain-growth polymerization, ${ }^{8}$ therefore a wide range of monomers are available for this type of modification. Even though this technique has been extensively used to modify the surface of polymers, during the last years it has also been extensively used to change the surface chemistry of nanoparticles such as cellulose nanofibers, ${ }^{11,12}$ carbon nanofibers and graphene platelets, ${ }^{13}$ zinc oxide, ${ }^{14}$ nanoclays ${ }^{15}$ and titanium oxide nanoparticles. ${ }^{16}$ Although some previous reports have studied the surface modification of cellulose nanocrystals by this method, ${ }^{17}$ there is very few information in the literature regarding the versatility of the technique and the application in incorporation to polymer matrixes.

The objective of this study is to evaluate the plasma-induced polymerization as an alternative to modify the surface of 
cellulose nanocrystals, utilizing different monomers of different nature: styrene, caprolactone and farnesene. The surface characteristics of the nanocrystals were studied by different techniques including XPS, FTIR and STEM, demonstrating the successful and homogenous modification, which did not alter the rod-like shape of the nanocrystals, thus their anisotropy. The employment of the modified nanocrystals as reinforcement additive of ABS was also studied utilizing polycaprolactonedeposited CNC's as model system. The incorporation of modified CNC's in ABS via in situ polymerization significantly enhanced the impact resistance of the thermoplastic, which could have great implications for industrial applications.

\section{Experimental part}

\section{Materials}

High-purity cellulose from softwood fibres (Norwegian spruce) with high cellulose content (95\% cellulose, $4.5 \%$ hemicellulose and $0.1 \%$ lignin content) were used as feedstock of cellulose nanocrystals, and were used as provided by Domsjö Fabriker $\mathrm{AB}$, Sweden. Styrene (4-tert-butylcatechol as stabilizer, $\geq 99 \%$ ), acrylonitrile (35-45 ppm monomethyl ether hydroquinone as inhibitor, $\geq 99 \%$ ), 2,5-dimethyl-2,5-di-(2-ethylhexanoylperoxy) hexane (Luperox 256), (DI-tert-butyl peroxide 98\%), tert-dodecylmercaptan (TDM), tert-butyl perbenzoate and $\varepsilon$-caprolactone (97\%) were purchased from Sigma-Aldrich and used as received. Farnesene (trans- $\beta$-farnesene $\geq 95 \%$ ) was supplied by Amyris, and was distilled from sodium at reduced pressure before use. Commercial polybutadiene $\left[M_{\mathrm{w}}=750 \mathrm{~kg}\right.$ mol; 1,4 -cis $=88 \%$ and 1,2-vinyl $=11 \%$; PDI $=2.03]$ was used.

\section{Methods}

Extraction of cellulose nanocrystals (CNC's). The cellulose nanocrystals (CNC) suspension was prepared by sulphuric acid hydrolysis of cellulose pulp with $64 \% \mathrm{w} / \mathrm{w}$ sulphuric acid at $45{ }^{\circ} \mathrm{C}$ under vigorous stirring, following the process reported by Bondeson et al. ${ }^{18}$ The hydrolysis was terminated by quenching through adding an excess of distilled water after 45 minutes of reaction and cool down to room temperature. The suspension was repeatedly centrifuged at $4000 \mathrm{rpm}$ for $20 \mathrm{~min}$ to concentrate the cellulose nanocrystals and to remove the excess of aqueous acid. The resultant nanocrystals were then dialyzed against deionized water for 3 days until constant neutral $\mathrm{pH}$. The suspension was sonicated using a Qsonica Q500, 500 watt Sonicator at $75 \%$ output for 2 minutes, and freeze-dried prior modification.

Plasma-surface modification of cellulose nanocrystals. Plasma-induced polymerization was carried out based on the experimental procedures described by Neira et al. ${ }^{13}$ using a custom-made plasma reactor (see Fig. 1) consisting in 3 main parts: (i) reaction chamber: a $500 \mathrm{~mL}$ Pyrex glass flask where the reaction takes place, connected to a rotation evaporator (which acts as a bridge between the multiple components of the reactor and as agitation system), (ii) monomer gas delivery system: a Schlenk tube containing the monomer, connected to the main body of the rotary evaporator through a hose and a regulated gas

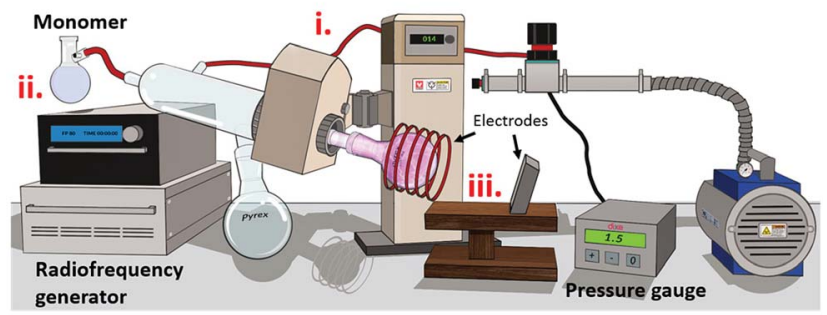

Fig. 1 Schematic representation of the custom-made plasma reactor used for CNC's surface modification.

valve. The monomer flow is obtained as a result of the vacuum in the system (acquired with a vacuum pump connected to the rotary evaporator); (iii) electrical excitation system: the electromagnetic field is obtained thanks to a copper wire, acting as an electrode, coiled around the glass flask with one end connected to a radiofrequency generator (Advance Energy RFX600/13.56 $\mathrm{MHz}$ ). For every modification, $1 \mathrm{~g}$ of freeze-dried CNC's was deposited into the glass flask and was put under vacuum until to achieve a pressure of $1.5 \times 10^{-1}$ mbar. After the base pressure was reached, the corresponding monomer was introduced and allowed to flow until reach a stable pressure of $2.5 \times 10^{-1} \mathrm{mbar}$ inside the reactor. Later, the plasma source was induced with the radiofrequency generator $(13.56 \mathrm{MHz}$ at a plasma power of $70 \mathrm{~W}$ ). Nanoparticles were treated under the previously stated conditions for 1 hour at stirring rates of $15 \mathrm{rpm}$.

Synthesis of ABS nanocomposites via in situ polymerization. Three different samples of ABS were synthetized, each one containing $0.5 \mathrm{wt} \%$ of CNC's: (1) a reference sample without CNC's (ABS), (2) a sample with unmodified CNC's (ABS/CNC); and (3) ABS with CNC's modified with polycaprolactone (ABS/ $\mathrm{CNC}_{\mathrm{Ca}}$ ). The utilized CNC's amount was decided based on previously reported works by El Miri et al. ${ }^{19}$ and Zhang et al. ${ }^{20}$ The synthesis of ABS nanocomposites was carried out via bulk polymerization in a stainless steel reactor (1 L capacity), equipped with an anchor-turbine stirrer and at room temperature. The reaction steps were as follows: (i) $14.4 \mathrm{~g}$ of polybutadiene (previously cut into small pieces), $143.66 \mathrm{~g}$ of styrene and $41.94 \mathrm{~g}$ of acrylonitrile were introduced into the reactor and let for stirring overnight at a rate of $45 \mathrm{rpm}$ in order to ensure total rubber dissolution. (ii) The CNC's were then introduced into the reactor and dispersed in the monomer solution via ultrasonication for 30 minutes at an amplitude of $40 \%$. The temperature and stirring rate were then fixed at $90{ }^{\circ} \mathrm{C}$ and $100 \mathrm{rpm}$ respectively, followed by the addition of Luperox 256 $(0.05 \mathrm{~g})$ and tert-dodecylmercaptan $(0.2 \mathrm{~g})$. The conditions were kept constant until the final conversion reached $\geq 35 \%$ (ensuring the phase inversion phenomenon). Subsequently, tert-butyl perbenzoate $(0.2 \mathrm{~g})$ was added to the reaction mixture. (iii) Finally, the reaction mixture was introduced into glass containers and left at $150{ }^{\circ} \mathrm{C}$ for 17 hours until the polymerization was carried out up to total monomer conversion. The resulting product was milled and injected moulded for further characterization. A schematic diagram for the synthesis of ABS/ CNC nanocomposites is shown in Fig. 2. 


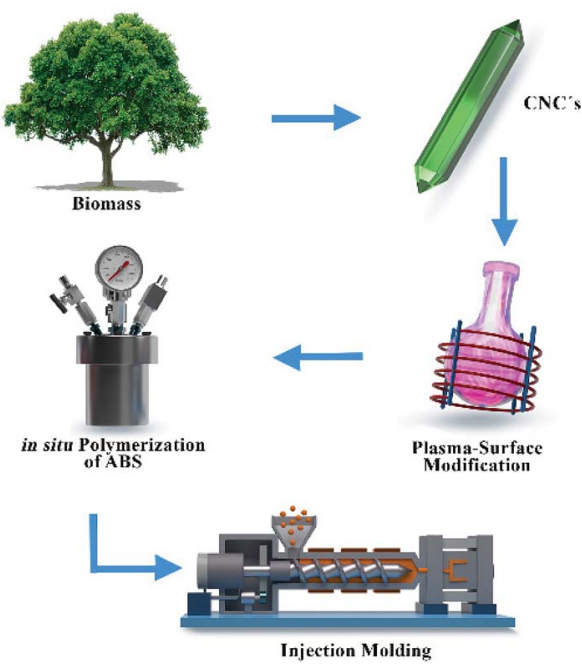

Fig. 2 Schematic diagram of the process followed for the surface modification of nanocrystals and the synthesis of ABS/CNC nanocomposites.

Injection moulding. For each sample, the milled material was melted using a lab scale co-rotating twin-screw extruder (Xplore micro compounder $15 \mathrm{~mL}$ ) at a barrel temperature of $200{ }^{\circ} \mathrm{C}$ for 2 and a half minutes. Thereafter, the injection unit (Xplore micro-injection moulder IM 12) was filled with the melted polymer directly from the micro compounder and injected into the mould cavity (with a maintained temperature of $28{ }^{\circ} \mathrm{C}$ ) at a pressure of $12 \mathrm{kN}$. Each cycle of the process lasts approximately 40 seconds.

\section{Characterization}

Surface-modified cellulose nanocrystals. The thermal behaviour of the nanocellulose samples was assessed by thermogravimetric analyses (TGA) using a thermoanalyser TA 2000 for measuring the transformation in mass of the samples a function of time in an interval of $30-600{ }^{\circ} \mathrm{C}$ at a heating rate of $5 \mathrm{~K} \mathrm{~min}^{-1}$ under nitrogen gas at a flow rate of $20 \mathrm{~mL} \mathrm{~min}^{-1}$. The thermal transitions of the cellulose nanocrystals was studied by Differential Scanning Calorimeter (DSC) using a Netzsch 214 Polyma DSC at a heating rate of $5 \mathrm{~K} \mathrm{~min}^{-1}$. The morphology and topography of the samples was assessed by Scanning Transmission Electron Microscope (STEM) conducted on a Jeol JEM $2100 \mathrm{~F}$ with accelerating voltage of $80 \mathrm{kV}$. For analysis, a droplet of diluted suspension of the sample was deposited on a carbon microgrid prior examination and allowed to dry. The chemical composition of the nanocrystals was analysed by FourierTransform Infrared-Spectroscopy (FTIR) using a ThermoScientific FTIR/ATR spectrometer, model Nicolet iS50. The spectra were the result of 32-times averaged scans and were acquired at a resolution of $4 \mathrm{~cm}^{-1}$ from 500 to $4000 \mathrm{~cm}^{-1}$. X-ray photoelectron spectroscopy was used to determine the chemical structure of the nanocrystals upon modification. The analyses were collected with a PHI VersaProbe II using a monochromatized $\mathrm{A} 1 \mathrm{~K} \alpha$ radiation operating at $150 \mathrm{~W}$ and energy of $20 \mathrm{eV}$ for individual photoelectron lines. All the binding energies were referenced to the $\mathrm{C}$ 1s hydrocarbon peak at $284.6 \mathrm{eV}$. The high-resolution C 1s spectrum was fitted using a Shirley background subtraction and a series of Voigt peaks. The crystallinity degree of the nanocrystals was measured via powder X-ray diffraction (PXRD) using a PANalytical X'Pert PRO $\mathrm{X}$-ray system with the settings viz. current $40 \mathrm{~mA}$, tension of 45 $\mathrm{kV}$, at room temperature with step size of 0.05 . The calculation of the crystallinity degree of the nanocrystals was estimated using the deconvolution of the PXRD spectra method.

ABS nanocomposites. The molecular weight of the ABS samples was determined by size exclusion chromatography (SEC) conducted on an Agilent PL-GPC 50 System, using polystyrene standards. THF (HPLC grade) as eluent at $40{ }^{\circ} \mathrm{C}$. The morphology of the ABS samples was examined using a Transmission electron microscopy (TEM) TITAN 80-300 kV. Samples were prepared by ultramicrotomy technique and stained with osmium tetroxide $\left(\mathrm{OsO}_{4}\right)$ vapours. The soluble fraction was precipitated from methanol and both fractions were dried under vacuum at $50{ }^{\circ} \mathrm{C}$ to constant weight and gravimetrically calculated. The gel-\% was calculated by separating the gel fraction (insoluble) from the soluble fraction (free SAN), by dissolving $1 \mathrm{~g}$ of a polymerized ABS sample in $25 \mathrm{~mL}$ of a toluene/thf (50/50 solution) during $25 \mathrm{~h}$. After that, the swollen gel was centrifuged for 1 hour at $20000 \mathrm{rpm}$ and at $-20{ }^{\circ} \mathrm{C}$, in order to separate the two phases. The swelling index was determined as the ratio of the weight of swollen gel to that of the dry gel. The viscoelastic properties of the samples were measured on injection-molded specimens on a Dynamic Mechanical Analyzer (TA Instrument), in flexural mode from $-140{ }^{\circ} \mathrm{C}$ to $110^{\circ} \mathrm{C}$, using a frequency of $0.1 \mathrm{~Hz}$, an amplitude of $0.5 \mathrm{~mm}$, and a heating rate of $5^{\circ} \mathrm{C} \mathrm{min}^{-1}$. The impact strength was measured based on ASTM D256, using the Izod Impact Testing (Notched Izod) standard, where the sample is clamped into the pendulum impact test fixture with the notched side facing the striking edge of the pendulum.

\section{Results and discussion}

In this work, we modified the surface of cellulose nanocrystals via plasma-surface modification using a variety of monomer units (see Fig. 3) of different nature: caprolactone $\left(\mathrm{CNC}_{\mathrm{Ca}}\right)$, styrene $\left(\mathrm{CNC}_{\mathrm{St}}\right)$, and farnesene $\left(\mathrm{CNC}_{\mathrm{Fa}}\right)$, to produce a polyester, polyolefin and polyterpene respectively.

The modification was done in order to enhance the dispersion and compatibility with hydrophobic polymer matrixes of cellulose nanocrystals, proving at the same time the versatility of plasma-induced modification technique. In addition, the CNC's modified with polycaprolactone $\left(\mathrm{CNC}_{\mathrm{Ca}}\right)$ were selected as
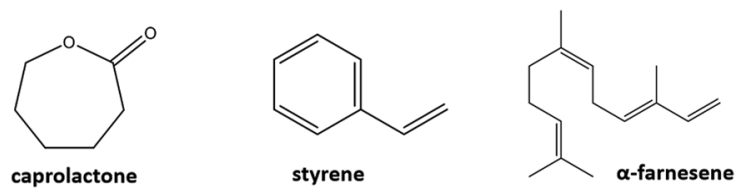

Fig. 3 Different monomers used for the surface functionalization of cellulose nanocrystals. 
model system to be tested as reinforcement additive of ABS nanocomposites. In plasma-induced polymerization, a strong electrical field ionizes and excites molecules and radicals, which then bombard the surface of the nanocrystals, inducing a chains growth on the surface via plasma deposition. ${ }^{8}$ A schematic representation of the custom-made plasma reactor used for the surface modification is shown in Fig. 1. As previously detailed in the experimental part, the reaction takes place in a flask, connected to a vacuum system which induces a flow in gas phase through the reaction chamber. Simultaneously, a radiofrequency generator electrically excites the medium, and generates plasma for its subsequent polymerization ${ }^{\mathbf{1 0}}$ (real photographs of setup in Fig. S1†).

\section{Characterization of modified cellulose nanocrystals}

The successful functionalization of CNC's was confirmed analyzing their chemical composition by FTIR spectroscopy using the ATR (Attenuated Total Reflection) technique, and results are shown in Fig. 4a (full FTIR spectra in Fig. S3†). Overall, the characteristic bands of saccharides, including the peaks at 2893 and $1400 \mathrm{~cm}^{-1}$ corresponding to the $\mathrm{C}-\mathrm{H}$ stretching bands and bending of $-\mathrm{CH}_{2}$ groups, remain unchanged. $^{2,21}$ No significant change in the $\mathrm{OH}$ signal at $3300 \mathrm{~cm}^{-1}$ was observed, as the modification only affected the surface hydroxyl groups, while the internal groups of cellulose remained untouched. ${ }^{22}$ However, all modified nanocrystals, showed the appearance of a small peak in the range of $1700 \mathrm{~cm}^{-1}$ (highlighted in Fig. 4a), which presumably indicates the contribution from the deposited polymers, for instance suggest the carboxyl group of the caprolactone structure in the case of $\mathrm{CNC}_{\mathrm{Ca}}{ }^{23}$ the stretching frequency of the isolated double bond in the structure of polyfarnesene for $\mathrm{CNC}_{\mathrm{Fa}}{ }^{24}$ and one of the overtone of the aromatic ring of polystyrene ${ }^{25}$ for $\mathrm{CNC}_{\mathrm{St}}$. No other characteristic peaks corresponding to complementary functionalities could be distinguished due to the interference with cellulose peaks which predominate in the spectra.
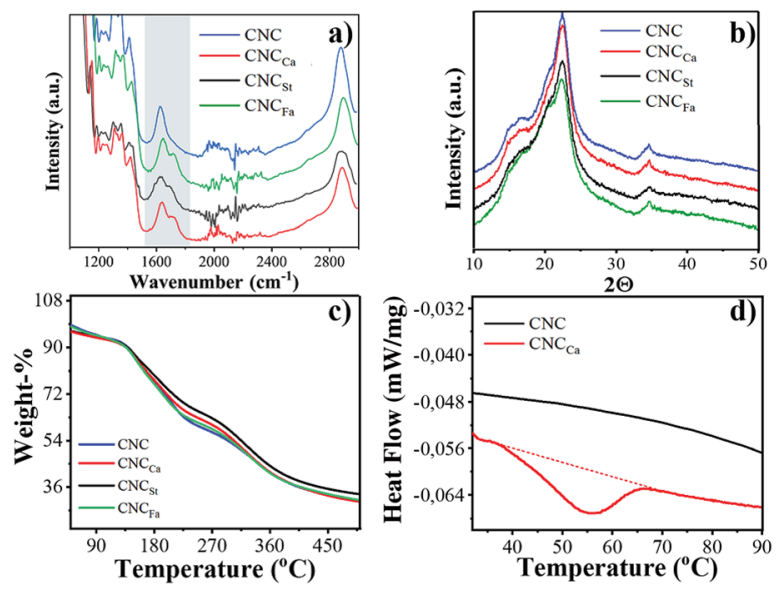

Fig. 4 (a) FTIR spectra; (b) X-ray diffraction (XRD) pattern of untreated and modified CNC's; (c) TGA thermogram of the modified cellulose nanocrystals, and (d) Differential Scanning Calorimetry (DSC) analysis of $\mathrm{CNC}$ and $\mathrm{CNC}_{\mathrm{Ca}}$.
The influence of surface coating over the thermal degradation behaviour of the modified CNC's, was studied by means of thermogravimetric analysis (TGA) and the results are presented in Fig. 4c. The thermogram show that the modified nanocrystals exhibit a decrease in weight loss-\% at $250{ }^{\circ} \mathrm{C}$ (the onset of degradation of cellulose), attributed to the presence of a substantial polymer coat on the surface of the nanocrystals, which degrades at slightly higher temperatures than cellulose due to their aliphatic nature. Consequently, this corroborates the successful modification by this technique. The deposition of the amorphous polymer coating layer was also reflected as a decrease in the crystallinity degree of the cellulose nanocrystals, studied by PXRD (see Fig. 4b). The crystallinity degree of the pristine nanocrystal (70\%) decreased to 46.39, 50.07 and $56.33 \%$ corresponding to $\mathrm{CNC}_{\mathrm{St}}, \mathrm{CNC}_{\mathrm{Ca}}$ and $\mathrm{CNC}_{\mathrm{Fa}}$ respectively.

The size and topography of the nanocrystals was studied by high-resolution Scanning Transmission Electron Microscopy (STEM) and the results are shown in Fig. 5. In general, all CNC's exhibited a typical rod-like structure, which implies that the modification does not alter the anisotropic behavior that is characteristic of the nanocrystals. The pristine CNC exhibited an average cross- sectional dimension of $14.7 \pm 2.9 \mathrm{~nm}$, which slightly increased after modification due to the deposition of the polymer on the surface to $15.22 \pm 1.3 \mathrm{~nm}\left(\mathrm{CNC}_{\mathrm{st}}\right), 24.15 \pm$ $3.0\left(\mathrm{CNC}_{\mathrm{Ca}}\right)$, and $19.13 \pm 3.6 \mathrm{~nm}\left(\mathrm{CNC}_{\mathrm{Fa}}\right)$. The presence of the polymer layer was appreciated as spherical structures decorating the surface of the nanocrystals (see Fig. 5), proving that they were densely and homogenously modified. Further micrographs of the modified nanocrystals are shown in Fig. S4. $\dagger$

$\mathrm{X}$-ray photoelectron spectroscopy (XPS) was used to study the surface chemistry of the cellulose nanocrystals upon modification, and the results are shown in Fig. 6 and Table 1. The chemical composition, as can see from the XPS survey spectra (see Fig. S5 $\dagger$ ) display a significant increase in the carbon atomic percent upon modification (see Table 1), due to the aliphatic structure of the polymer layer. Nevertheless, coating of polycaprolactone $\left(\mathrm{CNC}_{\mathrm{Ca}}\right)$ gave place to a lower $\mathrm{C} / \mathrm{O}$ ratio compared to the other monomers, due to the polyester structure of
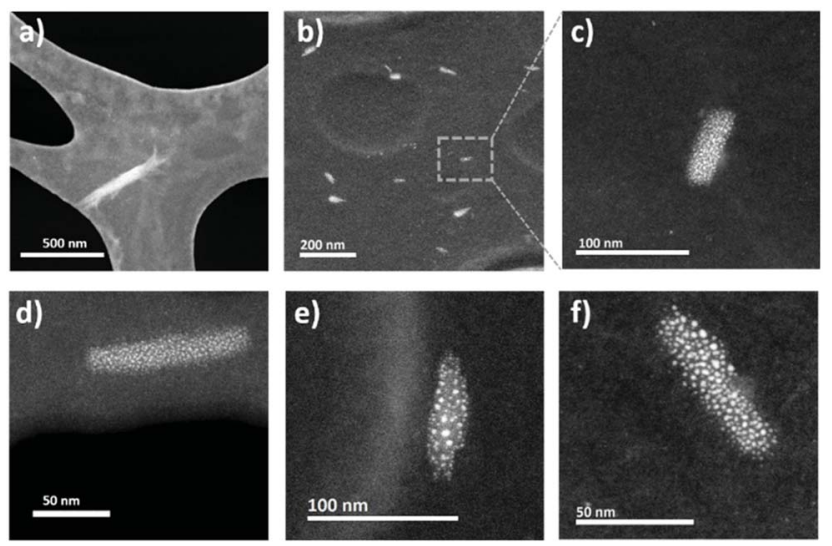

Fig. 5 Surface topography of the modified cellulose nanocrystals studied by STEM: (a) CNC; (b and c) $\mathrm{CNC}_{\mathrm{Ca}}$; (d) $\mathrm{CNC}_{\mathrm{St}}$; and (e and f) $\mathrm{CNC}_{\mathrm{Fa}}$. 

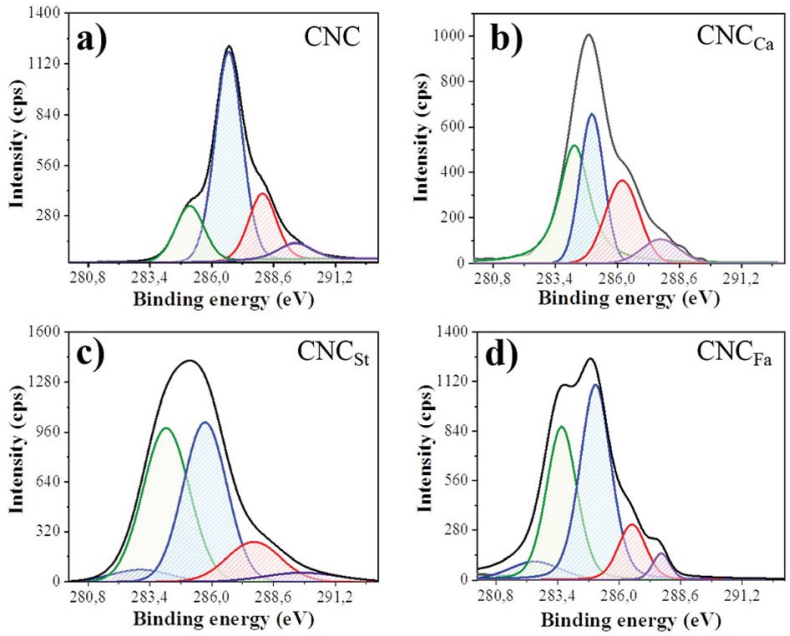

Fig. 6 Peak-fitted high-resolution C1s XPS spectra of the (un)modified cellulose nanocrystals.

Table 1 Surface composition of the modified nanocrystals studied by X-ray photoelectron spectroscopy (XPS) and the C 1s high-resolution data

\begin{tabular}{lllllll}
\hline \multicolumn{7}{c}{ Binding energy ${ }^{b}(\mathrm{eV})$} \\
\cline { 3 - 7 } Sample & ${\mathrm{C} / \mathrm{O}^{a}}^{c}$ & 284 & $\begin{array}{l}285.5 \\
(\mathrm{C} 1)\end{array}$ & $287(\mathrm{C} 2)$ & $288(\mathrm{C} 3)$ & $289(\mathrm{C} 4)$ \\
\hline $\mathrm{CNC}$ & 1.38 & - & $10.9 \%$ & $70.7 \%$ & $10.0 \%$ & $2.2 \%$ \\
$\mathrm{CNC}_{\mathrm{St}}$ & 4.40 & $5.10 \%$ & $40.8 \%$ & $37.2 \%$ & $13.5 \%$ & $3.4 \%$ \\
$\mathrm{CNC}_{\mathrm{Ca}}$ & 2.06 & - & $39.3 \%$ & $29.8 \%$ & $23.3 \%$ & $7.5 \%$ \\
$\mathrm{CNC}_{\mathrm{Fa}}$ & 5.54 & $11.7 \%$ & $40.9 \%$ & $32.6 \%$ & $10.3 \%$ & $4.3 \%$
\end{tabular}

${ }^{a}$ Determined from XPS survey spectra (see ESI). ${ }^{b}$ Calculated from the deconvoluted peak-fitted $\mathrm{C} 1 \mathrm{~s}$ high resolution data.

polycaprolactone, which includes an oxygen atom in its composition.

The deconvoluted high-resolution C 1s spectra (see Fig. 6) of the nanocrystals suggests three main chemical environments of carbon, as reported before for cellulosic materials: one major peak at $286.8 \mathrm{eV}$ corresponding to C-O bond; a peak at $285 \mathrm{eV}$ corresponding to $\mathrm{C}-\mathrm{C}$ bonds, and a third one around $288 \mathrm{eV}$ that corresponds to $\mathrm{O}-\mathrm{C}-\mathrm{O}$ bond. ${ }^{26}$ Furthermore, a fourth small population around $289 \mathrm{eV}$ was also appreciated, corresponding to the presence of carboxyl groups, present before and after modification. In order to elucidate the surface modification carried out in this work, the attention was centered specially in the aliphatic carbon region designated as C1 (centered at 285 $\mathrm{eV}$ ), as normally cellulose is devoid of C1 carbon because of its polysaccharide structure. ${ }^{2}$ Therefore, the significant increase in C1 structure (see Fig. 6) confirms the presence of the polymer deposited over the surface of the nanocrystals, and the deconvoluted integral areas of each chemical state of carbon in the $\mathrm{C}$ 1s spectra, presented in Table 1, gives an approximate notion of the amount of polymer over the surface of the nanocrystals. It's noteworthy that the unmodified cellulose nanocrystals (CNC) also showed a small population of $\mathrm{C} 1$ carbon, which is suggested to correspond to residual lignin in the polysaccharide structure. ${ }^{2}$ Moreover, the nanocrystals modified with polystyrene and polyfarnesene $\left(\mathrm{CNC}_{\mathrm{St}} \& \mathrm{CNC}_{\mathrm{Fa}}\right)$ displayed the presence of an additional signal around $284 \mathrm{eV}$, which corresponds to the $\mathrm{sp}^{2}$ bonding present in both polymers, as in the aromatic ring of polystyrene, and the conjugated diene structure of polyfarnesene. ${ }^{27}$

\section{Synthesis of ABS/CNC nanocomposites}

The application of the surface-modified nanocrystals as reinforcement additive of ABS (acrylonitrile-butadiene-styrene) nanocomposites was explored. ABS is a terpolymer made by polymerizing styrene and acrylonitrile in the presence of polybutadiene. ${ }^{28} \mathrm{ABS}$ is a very important thermoplastic polymer which is used in a wide range of applications covering from automotive parts, medical devices, to electrical and electronic assemblies, among others. The versatility of ABS arises mainly from the capacity to be injection moulded and extruded, together with the good acid resistance and advantageous mechanical properties. ${ }^{28}$ The good mechanical properties of ABS, compared to conventional polystyrene, are due to the presence of polar nitrile groups (from acrylonitrile) which polarity increases the intermolecular interaction among the chains; and due to the homogenously distributed rubber particles throughout the polymer matrix, which provides toughness even at low temperatures. ${ }^{29}$

For the preparation of the ABS nanocomposites, we used only the nanocrystals modified with caprolactone $\left(\mathrm{CNC}_{\mathrm{Ca}}\right)$ at a single concentration, as model system, chosen simply due to the biodegradable nature of polycaprolactone which goes in good agreement with the green/sustainable advantage of plasma- modification technique. The thermal transitions of the CNC's before and after modification were studied by Differential Scanning Calorimetry (see Fig. 4d), showing that the melting peak of polycaprolactone could be observed in $\mathrm{CNC}_{\mathrm{Ca}}$ around $55{ }^{\circ} \mathrm{C}$, as a definitive proof of the successful modification of CNC's, opposite to pristine nanocellulose which did not display any thermal transition, as expected.

The synthesis of ABS was carried out using the bulk polymerization technique, and the resultant polymers were injection-molded for further characterization (see Fig. S6 $\dagger$ ). The main properties of the ABS/CNC nanocomposites are presented in Table 2. The morphology of the synthesized ABS was studied by Transmission Electron Microscopy (TEM) and the corresponding micrographs are shown in Fig. 7c-e. All ABS exhibited a conventional "salami-like" structure, typical of these heterogeneous materials. In the micrographs: the white phase corresponds to the continuous phase of SAN, while the dark region corresponds to rubber particles (in the range of $1 \mu \mathrm{m}$ ) of polybutadiene embedded in the SAN copolymer matrix. Upon the incorporation of the modified $\mathrm{CNC}$ 's to the $\mathrm{ABS},\left(\mathrm{ABS} / \mathrm{CNC}_{\mathrm{Ca}}\right)$ an increment in the elastomeric phase (according to the gel value reported in Table 2) was observed, accompanied with irregularities in the morphology, as it can be observed in Fig. 7e. These irregularities are suggested to have taken place due to a decrease in the molecular weight of the SAN copolymers, (see 
Table 2 Main characteristics of the ABS/CNC nanocomposites

\begin{tabular}{|c|c|c|c|c|c|c|}
\hline Sample $^{a}$ & $M_{\mathrm{w}}{ }^{b}\left(\mathrm{~g} \mathrm{~mol}^{-1}\right) \times 10^{-4}$ & $\mathrm{PD}^{c}$ & $T_{\mathrm{g}}{ }^{d}\left({ }^{\circ} \mathrm{C}\right)$ & Gel (\%) & Swelling index (\%) & $\operatorname{Impact~strength~}^{e}$ (ft-lb per inch) \\
\hline ABS & 41.31 & 2.72 & 111.29 & 9.83 & 12.94 & $1.9 \pm 0.1$ \\
\hline $\mathrm{ABS} / \mathrm{CNC}$ & 48.39 & 2.15 & 95.78 & 12.12 & 11.58 & $2.5 \pm 0.2$ \\
\hline
\end{tabular}

${ }^{a} 7.2 \mathrm{wt} \%$ of polybutadiene was used in all cases. ${ }^{b}$ Molecular weight of SAN determined by Size Exclusion Chromatography (SEC). ${ }^{c}$ Polydispersity $\left(M_{\mathrm{w}} / M_{\mathrm{n}}\right)$ of SAN determined by SEC. ${ }^{d}$ Glass transition temperature determined by dynamic mechanical analysis. ${ }^{e}$ Impact strength measured by Izod Impact Testing.
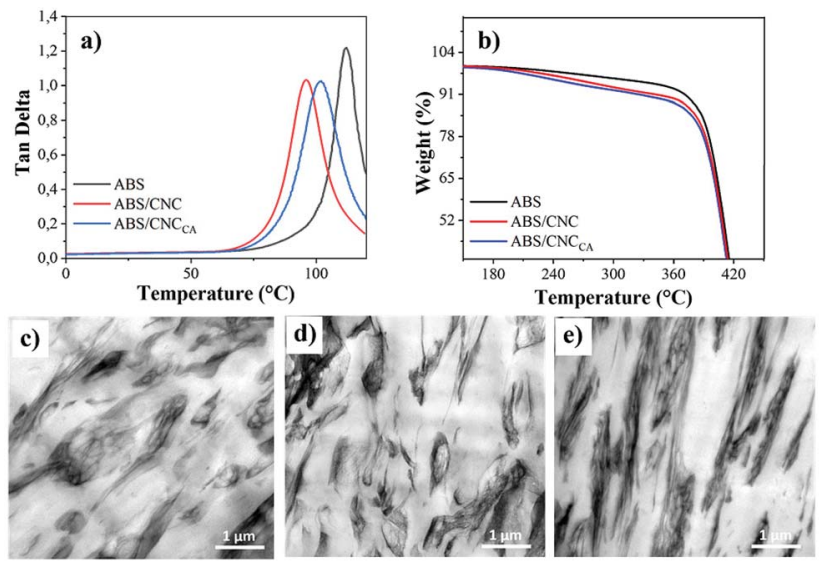

Fig. 7 Physical properties of ABS nanocomposites: (a) $\tan \delta$ of the ABS nanocomposites calculated from DMA; (b) TGA curves of ABS nanocomposites; (c-e) TEM micrographs of ABS: (c) ABS; (d) ABS/CNC and (e) $\mathrm{ABS} / \mathrm{CNC}_{\mathrm{Ca}}$.

Table 2), leading to a variation in the interfacial tension between the rubber phase and the SAN, as well as the viscosity ratio among the two phases, which are the two main parameters that establish the size and shape of rubber particles in ABS. ${ }^{30}$

The influence of the nanocrystals over the glass transition temperature $\left(T_{\mathrm{g}}\right)$ of $\mathrm{ABS}$ was investigated from dynamic mechanical analysis (see Fig. 7a and Table 2). The loss factor or $\tan \delta$ (ratio of loss modulus and storage modulus), which is a characteristic indicator of how much energy dissipates in the polymer matrix, was used as an indication of the $T_{\mathrm{g}}$ of the synthesized materials, as its peak maximum occurs at the transition regime from polymer's glassy to rubbery state. ${ }^{31}$ The pure ABS sample exhibited a glass transition of the SAN phase around $111{ }^{\circ} \mathrm{C}$, which shifted to lower temperatures upon loading of nanocrystals in the nanocomposites $\left(95.78{ }^{\circ} \mathrm{C}\right.$ for $\mathrm{ABS} / \mathrm{CNC}$; and $102{ }^{\circ} \mathrm{C}$ for $\mathrm{ABS} / \mathrm{CNC}_{\mathrm{Ca}}$ ). We suggest that the pristine $\mathrm{ABS}$ exhibits a higher $T_{\mathrm{g}}$ mainly due to the significantly lower Gel content- $\%$ compared to the composite ones. On the other hand, $\mathrm{ABS} / \mathrm{CNC}_{\mathrm{Ca}}$, even though it has lower molecular weight and a higher Gel content- $\%$ in comparison to ABS/CNC, it exhibits a higher $T_{\mathrm{g}}$ due to the significantly higher crosslinking degree of the elastomeric phase, which is reflected by swelling index value, reported in Table 2 . The thermal stability of the ABS composites was furthermore investigated by TGA, and the results are displayed in Fig. 7b. Overall, the degradation profile of all samples was seen as a sharp weight loss occurred around $400{ }^{\circ} \mathrm{C}$, which is the characteristic behavior of ABS. ${ }^{31}$ Nevertheless, the ABS/CNC nanocomposites exhibited a small degradation step at lower temperatures as well, corresponding to the contribution of cellulose nanocrystals, which degrade at lower temperatures $\left(\approx 250^{\circ} \mathrm{C}\right){ }^{2}$

The influence of the cellulose nanocrystals over the mechanical properties of ABS nanocomposites was studied, and the results are shown in Fig. 8 and Table 2. For most applications, the most important mechanical properties of ABS are impact resistance and toughness. In our system, it was observed that the incorporation of $\mathrm{CNC}_{\mathrm{Ca}}$ into $\mathrm{ABS}$, incremented in a $114 \%$ the impact resistance (see Table 2 and Fig. 8 b) compared to pure ABS. On the other hand, the ABS loaded with unmodified nanocrystals (ABS/CNC), also exhibited a higher impact strength than the reference material, but only in a $34 \%$. This enormous increase in mechanical performance could be attributed in part to the reinforcement of CNC's, which are known to possess outstanding mechanical properties. ${ }^{2}$ However, it is also known that the impact resistance in ABS is strongly dependent on the proportions of polybutadiene in relation to styrene-acrylonitrile (SAN). ${ }^{28}$ In our system, the incorporation of nanocrystals in the ABS had an influence in the molecular weight of the SAN phase, resulting in an increasing of the elastomeric phase (reflected in the gel-\% values in Table 2),

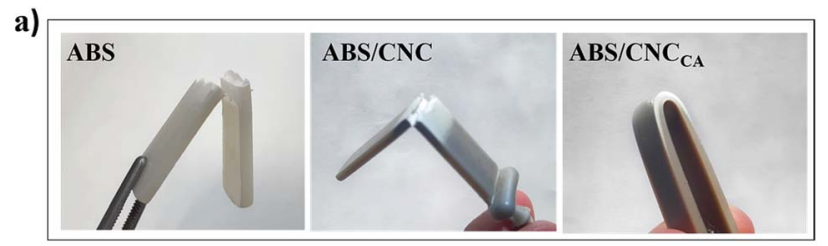

b)
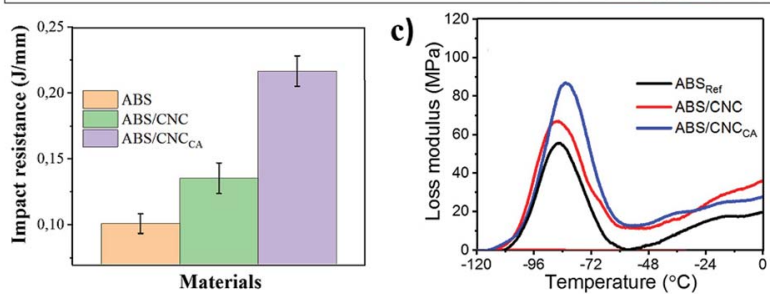

Fig. 8 Mechanical properties of ABS nanocomposites: (a) photographs of manual bent test applied to the injection molded samples displaying the mechanical reinforcement upon incorporation of modified nanocrystals; (b) impact resistance of ABS samples and (c) storage and loss modulus ABS nanocomposites measured by DMA. 
which is suggested to happen due to a possible interaction from the surface of the nanocrystals with the free radicals generated from the peroxide initiator employed for the synthesis of ABS.

The viscoelasticity of the materials after incorporation of the CNC's was analyzed from the Dynamic Mechanical Analysis (DMA) (see Fig. 7b, S9 and S10†), where a decrease in the integral area of the loss factor (tan delta) was observed upon incorporation of CNC's. As the loss factor indicates how much energy dissipates in the polymer matrix, a smaller value represents a more elastic behavior of ABS upon incorporation of the nanocrystals, compared to the reference material (see Fig. 7a). Moreover, the area under the loss modulus at the relaxation of the rubber phase at low temperatures increased when incorporation of the cellulose nanocrystals, especially upon plasma modification (see Fig. 8c). This area at lower temperatures corresponding to the loss modulus or tan delta can be affected by the volume fraction of the rubber phase (or gel phase) and therefore allows us to establish a relationship between the toughness and impact strength. In our case, the strength values ae consistent with the area under the loss modulus.

On the other hand, the storage modulus (see Fig. S9†) exhibited a different trend: the ABS/CNC composites demonstrated a higher modulus compared to the reference material, suggesting an enhanced stiffness of the material. Nevertheless, the $\mathrm{ABS} / \mathrm{CNC}_{\mathrm{Ca}}$ displayed a lower storage modulus, also attributed to the lower molecular weight of the SAN phase, which increments the rubber contribution, thus decreasing the elastic behavior of the resultant material.

\section{Conclusions}

We have demonstrated the applicability of plasma-induced polymerization as a versatile alternative for surface modification of cellulose nanocrystals, with three different monomers: styrene, caprolactone and farnesene. The modification did not alter the rod-like shape characteristic of the nanocrystals, and improved their compatibility with the polymer matrix. The application of the modified nanocrystals as a reinforcement additive of ABS was tested, showing that upon modification, a significant increase in the impact resistance of the thermoplastic was observed, which could have great implications for industrial applications.

\section{Conflicts of interest}

There are no conflicts to declare.

\section{Acknowledgements}

The authors acknowledge the financial support of the European Commission (MULTIMAT-H2020-MSCA-ITN-2014, Grant No. 676045) and the Mexican National Council of Science and Technology (CONACyT) (Grant 258278). The authors acknowledge the National Laboratory of Graphene Materials for the support to this research.

\section{References}

1 A. Dufresne, Philos. Trans. R. Soc., A, 2018, DOI: 10.1098/ rsta.2017.0040.

2 L. Valencia, V. Arumughan, B. Jalvo, H. J. Maria, S. Thomas and A. P. Mathew, ACS Omega, 2019, 4, 4330-4338.

3 D. Puglia, E. Fortunati and J. M. Kenny, Multifunctional Polymeric Nanocomposites Based on Cellulosic Reinforcements, William Andrew, 1st edn, 2016.

4 A. Carlmark, Macromol. Chem. Phys., 2013, 214, 1539-1544.

5 M. Tizzotti, A. Charlot, E. Fleury, M. Stenzel and J. Bernard, Macromol. Rapid Commun., 2010, 31, 1751-1772.

6 S. Hansson, E. Östmark, A. Carlmark and E. Malmström, ACS Appl. Mater. Interfaces, 2009, 1, 2651-2659.

7 Y. Yin, X. Tian, X. Jiang, H. Wang and W. Gao, Carbohydr. Polym., 2016, 142, 206-212.

$8 \mathrm{H}$. Yasuda and Y. Iriyama, in Handbook of Deposition Technologies for Films and Coatings, 2010, pp. 357-374.

9 P. Chu, Mater. Sci. Eng., R, 2002, 36, 143-206.

10 L. F. Ramos-de Valle, M. G. Neira-Velázquez and E. Hernández-Hernández, J. Appl. Polym. Sci., 2007, 107, 1893-1899.

11 T. Wakida, K. Takeda, I. Tanaka and T. Takagishi, Text. Res. J., 1989, 59, 49-53.

12 J. Felix, P. Gatenhol and H. P. Schreiber, Appl. Polym. Sci., 1994, 51, 285-295.

13 N.-V. M. Guadalupe, L. F. Ramos-de Valle, E. HernándezHernández and I. Zapata-González, e-Polym., 2008, 8, 162.

14 Y. de Rancourt, B. Couturaud, A. Mas and J. J. Robin, J. Colloid Interface Sci., 2013, 402, 320-326.

15 M. G. Neira-Velázquez, L. F. Ramos-Devalle, E. HernándezHernández, A. Ponce-Pedraza, S. G. Solís-Rosales, S. Sánchez-Valdez, P. Bartolo-Pérez and V. A. GonzálezGonzález, Plasma Processes Polym., 2011, 8, 842-849.

16 A. Solís-Gómez, M. G. Neira-Velázquez, J. Morales, M. A. Sánchez-Castillo and E. Pérez, Colloids Surf., A, 2014, 451, 66-74.

17 P. Samyn, M. P. Laborie, A. P. Mathew, A. Airoudj, H. Haidara and V. Roucoules, Langmuir, 2012, 28, 14271438.

18 D. Bondeson, A. Mathew and K. Oksman, Cellulose, 2006, 13, 171-180.

19 N. El Miri, K. Abdelouahdi, M. Zahouily, A. Fihri, A. Barakat, A. Solhy and M. El Achaby, J. Appl. Polym. Sci., 2015, 132, 113.

20 Y. Zhang, Y. Yang and K. Tang, Polym. Polym. Compos., 2007, 21, 449-456.

21 Z. Karim, A. P. Mathew, M. Grahn, J. Mouzon and K. Oksman, Carbohydr. Polym., 2014, 112, 668-676.

22 G. Morandi, L. Heath and W. Thielemans, Langmuir, 2009, 101, 8280-8286.

23 C. F. Bellani, E. Pollet, A. Hebraud, F. V. Pereira, G. Schlatter, L. Avérous, R. E. S. Bretas and M. C. Branciforti, J. Appl. Polym. Sci., 2016, 133, 4-11.

24 P. Sarkar and A. K. Bhowmick, RSC Adv., 2014, 4, 6134361354. 
25 D. Olmos and J. Gonza, Phys. Chem. Chem. Phys., 2014, 16, 24339-24349.

26 L. Valencia, S. Kumar, B. Jalvo, A. Mautner, G. SalazarAlvarez and A. P. Mathew, J. Mater. Chem. A, 2018, 6, 16361-16370.

27 C. Vannuffel, C. Vergnaud, A. Chabli, G. Rolland, M. N. Séméria, J. Baylet, A. Ermolieff, D. Rouchon and F. Pierre, Surf. Interface Anal., 2002, 31, 185-190.
28 J. D. Moore, Composites, 1973, 4, 118-130.

29 A. W. Birley, Handbook of Plastics and Elastomers, John Wiley \& Sons, Ltd, 1976, vol. 8.

30 C. Koning, M. Van Duin, C. Pagnoulle and R. Jerome, Prog. Polym. Sci., 1998, 23, 707-757.

31 X. Wei, D. Li, W. Jiang, Z. Gu, X. Wang, Z. Zhang and Z. Sun, Sci. Rep., 2015, 5, 1-7. 